\title{
APPROCHE ESTHÉTIQUE DE L'ORGANISATION : LE CAS DES SALLES DE CINÉMA
}

\author{
Florence Carion et Aurore Van de Winkel ${ }^{1}$
}

L'esthétique organisationnelle -concept émergent dans le champ de la communication des organisations- peut être considérée comme l'ensemble des éléments formels ou sensibles agencés dans un espace par une organisation. Ces éléments agiraient comme des signes ou des signaux auprès d'actants. L'esthétique aurait un impact sur les sens et la raison, elle se situerait au carrefour de l'intelligible et du sensible.

Ainsi définie, l'esthétique organisationnelle devrait pouvoir être appréhendée par l'approche des lieux mêmes où l'organisation se donne à voir, à sentir, à comprendre : les sites organisationnels.

Le domaine cinématographique constitue, dans cette optique, un lieu d'investigation particulier. Le monde du cinéma serait actuellement plus vivant que jamais ${ }^{2}$ et procurerait au spectateur un sentiment merveilleux d'évasion. Or, les complexes cinématographiques sont

1 Étudiantes du DEA en Communication, Département de communication, Université catholique de Louvain. Cet article a été rédigé sur base d'un travail de DEA réalisé par Enrique Aimone, Florence Carion, Fatima Oropesa Montes et Aurore Van de Winkel.

2 Un sondage, effectué en Communauté française, montre que le cinéma est le lieu de divertissement le plus fréquenté (J.-M. WYNANTS, "Cinéma, y aller ou pas ?", Le Soir, 26 janvier 2000). 
bien souvent décrits par la presse comme d'horribles monstres ${ }^{1}$, des mastodontes. L'apparition de ces sites imposants durcit surtout la concurrence face aux petits cinémas de quartiers. Nous sommes donc devant un secteur où les lieux organisationnels, très différents les uns des autres, vont essayer, chacun à sa manière, de faire ressentir des émotions aux spectateurs, tout en s'identifiant clairement en tant qu'organisations.

L'objectif de cet article est d'approcher l'esthétique des complexes cinématographiques en Belgique, par l'analyse des éléments concrets -relevés grâce à une démarche inductive d'observation et d'entretiens- qui, combinés, pourraient constituer ce phénomène selon notre définition. Pratiquement cette recherche porte sur les deux chaînes de cinémas les plus importantes de Belgique : les multinationales UGC et Kinepolis. Les observations ont été effectuées dans deux complexes de la Région flamande, deux complexes de la Région de Bruxelles-Capitale ainsi que dans deux cinémas de la Région wallonne. Nous avons également effectué une observation de référence dans un cinéma de quartier, le Ciné Centre de Rixensart. Une grille d'observation a été élaborée retraçant le parcours qui peut être effectué, par le public, dans le cinéma et épinglant les différents indices de l'expérience esthétique. Ces derniers ont été répartis selon qu'ils informent sur l'environnement du site, sur son "habillement" (architecture, identité visuelle...) ou sur son "habitation" (manière dont le site est habité par les différents actants). Afin de confronter nos observations, concernant les phénomènes de communication liés à l'esthétique organisationnelle, aux stratégies et politiques mises en œuvre par les deux organisations, nous avons procédé à des entretiens auprès des responsables de communication d'UGC et de Kinepolis Group. À partir de nos résultats, un retour sur la définition de l'esthétique organisationnelle sera effectué en vue de l'affiner afin qu'elle corresponde le mieux possible à la réalité empirique.

1 L. HonOREZ, "Distributions, exploitations, manifestations et révolutions!", Le Soir, 15 février 2000. 


\section{Du parking aux salles obscures : un parcours esthétisé}

\section{La chaîne française UGC' ou l'esthétique organisationnelle sobre}

Les complexes UGC (Anvers ${ }^{2}$, Bruxelles ${ }^{3}$ et Louvain-la-Neuve ${ }^{4}$ ) sont intégrés dans un environnement urbain. Les bâtiments sont rattachés aux immeubles avoisinants au cœur de la ville dans laquelle ils sont implantés. En effet, la chaîne estime que le cinéma ne doit pas être retranché en dehors de la cité, à la manière d'une "ciné-usine", mais qu'il a sa place dans les centres urbains.

Les façades -alliant le verre et le fer-et les halls d'entrée de ces cinémas sont principalement habillés par l'identité visuelle du groupe. Privilégiant la sobriété, UGC met en avant ses logos (sur les portes d'entrée et les banderoles d'accueil) et quelques publicités autopromotionnelles (dépliants UGC, affiches promouvant les modes alternatifs de réservations des places, pancartes présentant les projets du groupe...). Peu d'autres marques sont visibles dans ces espaces d'accueil, relativement dépouillés, qui servent également à l'organisation d'événements pour les particuliers ou les entreprises. Les messages communicationnels, et donc l'esthétique institutionnelle, concernent la chaîne elle-même et non les films proposés, qui semblent être présentés au second plan, sans logique de promotion de l'une ou l'autre production filmique particulière.

1 Premier groupe européen d'exploitation cinématographique, UGC s'est implanté en Belgique en 1978. La chaîne réalise $15 \%$ de parts de marché en Belgique.

2 Inauguré en mars 2001, l'UGC s'intègre dans un nouveau centre commercial d'Anvers, situé en plein centre-ville. Il est construit dans les locaux de l'ancien cinéma Gaumont.

3 En 1982, le groupe acquiert le cinéma "L'Eldorado" qui est rénové en 1992 et rebaptisé "UGC de Brouckère". UGC a effacé tout l'univers cubiste de l'ancien cinéma, ne laissant que l'architecture et les frises Art Déco à l'ambiance africaine de la célèbre grande salle classée, construite en 1938 et appelée, encore aujourd'hui, le grand Eldorado.

4 Il s'agit du plus grand site cinématographique du Brabant wallon. Il fait partie d'un projet regroupant également des activités de shopping et d'Horeca. La présence de cet UGC est vécue comme une chance économique par les autorités de la région et comme une participation à la "lutte contre la tendance à la désurbanisation" (M. BAUS, "UGC poursuit ses grandes manœuvres", La Libre Belgique, 26 septembre 2001). 
On trouve également au sein des bâtiments des indices d'une "valorisation de la culture cinématographique" (photographies d'acteurs, de réalisateurs, fresque, noms de réalisateurs donnés aux foyers...).

Malgré ces caractéristiques communes générales, il existe des différences évidentes entre les sites. En effet, deux de ces cinémas s'intègrent dans un projet plus global de centre commercial et de loisirs et présentent une identité visuelle commune (un logo coloré, entouré d'une ellipse bleutée) tandis que le troisième ne s'associe pas avec d'autres activités et est encore habillé de l'ancien logo (un simple agencement des lettres UGC sous forme de néons). Ce phénomène observable n'est que transitoire, les perspectives de la chaîne étant de continuer à créer des complexes intégrés dans des projets plus larges, représentatifs de l'état d'esprit actuel du groupe. Ce dernier se traduit par le développement du concept "Ciné Cité", un projet innovateur se démarquant des perspectives des chaînes concurrentes. Il consiste à augmenter la projection de films en version originale, à proposer une offre diversifiée de films de qualité (films français, polonais, asiatiques...) -qui peuvent être de grand public ou d'art et d'essai-au détriment des "blockbusters" américains. Mais le projet a également pour objectif de proposer des animations avant les films comme des conférences, des expositions (de photographies, de peintures...), des concerts. La chaîne veut faire du cinéma un véritable lieu de rencontre culturelle. Le spectateur est accueilli, peut visiter une exposition et se détendre dans des endroits chaleureux à l'ambiance "feutrée", comme les "ciné cafés". Par ce parcours, le spectateur pénétrerait dans un lieu possédant une dimension de rêve. Les sites UGC ne correspondant pas encore entièrement à cette image vont évoluer dans ce but.

Si le concept est clairement établi dans l'esprit de la direction du groupe, les résultats se font encore attendre sur le terrain. Le cinéma de Louvain-la-Neuve, censé représenter ce projet de la façon la plus aboutie, ne présente en effet pas ces caractéristiques de lieu à dimension de rêve ou particulièrement chaleureux. La décoration intérieure du bâtiment est relativement sobre -voire dépouillée- tandis que le "ciné café" tente de recréer une ambiance digne des bars américains qui détonne dans l'ensemble du cinéma. L'ambiance fonctionnelle met en évidence la dimension organisationnelle pure, marchande, plutôt que l'atmosphère d'évasion prônée. 


\section{La chaîne belge Kinepolis ${ }^{1}$ ou l'esthétique organisationnelle évincée}

Contrairement à UGC, les cinémas Kinepolis (Métropolis d'Anvers ${ }^{2}$, Kinepolis Bruxelles ${ }^{3}$, Imagibraine de Braine 1'Alleud ${ }^{4}$ ) n'ont pas d'appellation commune. Chaque nouveau site d'exploitation doit déjà avoir une certaine renommée, chaque complexe est nommé différemment selon la région dans laquelle il se situe, ce qui met en exergue son influence régionale. Cela montre une tentative d'intégration des sites au contexte environnemental mais traduit également un déficit d'identité commune. En effet, ce groupe est le fruit de l'association de deux constructeurs et exploitants de salles de cinéma désireux d'accéder à la cotation en bourse. Leurs deux identités et esthétiques organisationnelles distinctes et particulières ont donc dû être unifiées notamment par l'adoption du logo en forme d'étoile. Le groupe Kinepolis a ensuite dû développer une stratégie marketing, commerciale et communicationnelle commune à tous ses sites. Cette politique organisationnelle définit Kinepolis comme une "marketing driven company", une société qui est inspirée à tous niveaux, que ce soit au niveau de l'exploitation, au niveau de la communication ou au niveau de la vente, par une stratégie, une politique de marketing très approfondie, très développée. Cela se traduit concrètement dans le respect de quatre critères stratégiques qui garantissent le succès du groupe et fondent son identité : l'accessibilité au site -des parkings vastes et des routes d'accès nombreuses doivent permettre la mobilité autour des complexes-, un large choix de films et donc de salles, une

1 Le groupe Kinepolis, constructeur et exploitant de salles de cinéma ainsi que distributeur de films (Kinepolis Film Distribution), est le produit de l'association de la famille limbourgeoise Bert avec la famille Claeys, originaire de Flandre occidentale. C'est en partie grâce à ce groupe, enregistrant $50 \%$ du marché belge. que la Belgique a été le premier pays d'Europe à avoir été équipé de multiplexes puis de mégaplexes.

2 Avec ses 24 salles, le Metropolis d'Anvers était jusqu'à la construction du Kinepolis Madrid, en 1998, le plus grand cinéma du monde.

3 Le Kinepolis Bruxelles a été construit en 1988 au Heyzel sur l'ancien site de General Motors -ce qui explique la structure en spirale typique des grands parkings permettant d'aller d'un étage à l'autre du complexe.

4 Propriété d'une famille belge, l'Imagibraine de Braine l'Alleud a été repris pour $20 \%$ par le groupe Kinepolis. Son concept est en pleine évolution et se concrétisera par la construction d'un centre commercial annexé au cinéma. 
grande qualité de son et d'image ainsi qu'une disposition des salles assurant le confort du spectateur.

Influencé par ces quatre critères, l'agencement des bâtiments est similaire dans tous les sites. Les cinémas comprennent un vaste hall d'accueil éclairé par une façade vitrée. Les salles se répartissent de part et d'autre de deux longs couloirs sombres dans lesquels est diffusée une musique quelquefois adaptée aux grands événements de l'actualité (musique de Noël ou musique solennelle lors d'enterrements royaux par exemple). Le reste de la décoration intérieure est laissé au soin du gérant de chaque site, ce qui donne lieu à des variations d'éléments importants de l'identité Kinepolis (ainsi le logo peut varier en couleurs selon les lieux). Malgré le souci du groupe d'adaptation à l'environnement, l'architecture particulière des complexes n'est pas toujours appréciée ni du public, ni de la presse qui décrivent notamment Kinepolis Bruxelles comme un "ciné-ghetto" au look d'aérogare ou comme le "monstre de Brupark" extérieures au groupe telles que ces griefs urbanistiques influencent également la construction et l'aménagement des complexes.

Kinepolis Group met en exergue l'importance accordée à la qualité de projection des films -majoritairement américains-, à l'accueil et à la communication; ces éléments priment sur la décoration et l'aménagement des locaux. En effet, le groupe veut instaurer une certaine ambiance : aller voir un film au Kinepolis doit être aussi une occasion de voir du monde. Pour favoriser cela, toutes les séances commencent au même moment. Par souci de confort, les spectateurs sortent de la projection par des couloirs les ramenant vers le hall d'accueil et non pas par des sorties secondaires mal entretenues comme c'est le cas dans d'autres cinémas. Les décors sont amovibles, ce qui permet d'organiser des événements ponctuels, des animations. Jusqu'en 1997, la stratégie communicationnelle de Kinepolis était avant tout basée sur la communication interne, le bouche-à-oreille faisant le reste. Actuellement, les cinémas de la chaîne Kinepolis sont perçus commé évoluant vers des "leasure mails", des centres de loisirs où l'on peut également se sustenter et faire du fun-shopping. En effet, ce groupe s'allie à des partenaires très diversifiés tels les banques, les salons de coiffure, les magasins de musique, les fast-

1 A. JOASSIN, "Qui a peur du grand ciné-ghetto ?", Intermédiaire, n 7,11 février 1992, p. 13.

2 R. Callebaut, "Le syndrome Kinepolis donne des complexes", Univers Cité, $n^{\circ} 33$, février 1990 , p. 89. 
foods... et leur loue des emplacements sur son site. En cela, ces mégaplexes sont comparés à des hôtels cinq étoiles et aux sites du secteur aéroportuaire. À l'heure où la grande majorité des cinémas offrent la même qualité de son et d'image, le groupe veut se distinguer en privilégiant le renouvellement constant de la décoration et un développement des services destinés à différents publics : les jeunes (mise à disposition de playstations ou de connexions à internet) ou les familles (la "Kinecrèche"). Kinepolis devient donc un "Family Entertainment Center" qui veut véhiculer des valeurs comme la famille ou le loisir. Les entreprises ne sont pas oubliées puisque le groupe commence à installer des "Business and Communication Centers"'. L'importance du partenariat économique est frappante de par la présence de nombreux sponsors, d'objets et d'actions publicitaires au cœur des sites. La politique de la chaîne en la matière est souvent liée à celle des agences de publicité et cela se ressent dans l'habillement et l'habitation des complexes.

Pourtant, malgré les discours des responsables, il semblerait que les stratégies, concernant les manifestations tangibles du concept d'esthétique, n'aient été que peu abordées au sein de l'entreprise. Nous n'avons, en effet, jamais pu avoir une définition concrète et claire de ces dernières et les quelques indices vus plus haut nous permettent de croire que la maison-mère ne contrôle pas tous les éléments concernant son esthétique, beaucoup de détails étant laissés à la convenance des managers des sites. Le client peut alors difficilement reconnaître à l'intérieur des complexes des éléments d'identité visuelle du groupe. En fait, c'est la submersion de ces éléments, par les traces identitaires et marketing des partenaires économiques, qui permet de savoir que l'on se trouve bien dans un Kinepolis. De plus, malgré la revendication d'une identité européenne ${ }^{2}$ depuis son entrée en bourse en 1999 et son expansion dans des pays européens, le groupe met plus en avant son identité flamande ${ }^{3}$. La faible présence d'éléments identitaires au sein des différents complexes est liée à la

1 Des salles confortables équipées d'un matériel haute technologie de projection, de traduction et de connexion au net, permettent de recevoir les organisations lors de rencontres professionnelles.

2 G. RegelbrugGe, "Poussée expansionniste dans le paysage cinématographique", Média Marketing, $\mathrm{n}^{\circ} 158$, mai-juin 2000, p. 19.

3 Le néerlandais est devenu pratiquement la seule langue des publicités audiovisuelles ; Kinepolis sponsorise la production de films flamands... 
"business history" du groupe, par les problèmes des deux exploitants à s'accorder sur une identité commune.

Cette difficulté de ne pouvoir saisir le concept de l'esthétique générale du groupe a été expliquée par la notion d'innovation, le changement perpétuel qui étonne le spectateur et permet une remise en question de l'entreprise. Pourtant il ne s'agit, en fait, pour la plupart, que de modifications superficielles qui ne reflètent pas une véritable mutation du concept. Malgré les discours, il semble que Kinepolis axe encore sa stratégie organisationnelle sur la qualité de projection ainsi que sur le développement de services et non sur une esthétique travaillée en tant que telle.

\section{Les chaînes de cinéma : une esthétique commune ?}

\section{L'environnement et l'architecture}

Un premier élément de comparaison entre les deux chaînes est celui de l'architecture des bâtiments, directement liée à leur environnement. Les bâtiments de l'UGC sont intégrés dans un environnement urbain et sont rattachés aux immeubles les entourant. Les complexes du Kinepolis sont, par contre, situés dans un "no man's land", un endroit toujours relativement éloigné du centre des villes. À ce propos, le porte-parole du Kinepolis a déclaré en 2000: "Les complexes UGC et Kinepolis sont complémentaires car nos collègues français possèdent une fonction urbaine marquée tandis que la nôtre est davantage régionale" . L'architecture du Metropolis est, en effet, marquée par une volonté d'intégration et de référence aux caractéristiques locales de la ville portuaire d'Anvers (le bâtiment évoque un bateau avec des hublots...). Mais excepté ce site, les bâtiments du groupe Kinepolis ont une architecture relativement standardisée, peu liée au lieu d'implantation. Les cinémas de cette chaîne peuvent dès lors être rapprochés, dans une certaine mesure, de ce que Marc Augé appelle des "non-lieux", des espaces sans mémoire, que l'on retrouve, identiques, où que l'on aille dans le monde.

Les cinémas UGC, de par leur intégration dans les villes et leur aménagement, pour deux des trois sites observés, dans d'anciens cinémas dont ils portent parfois encore les traces, répondent moins à

I G. RegelbrugGe, op cit., p.19. 
ce modèle standard dans leur structure architecturale même si toutes leurs façades sont composées de parois de verre. Malgré tout, l'UGC Louvain-la-Neuve nous montre une évolution dans le concept architectural : la construction d'un bâtiment sur un site vierge permet aux concepteurs de donner libre cours à leur vision des choses; la façade rappelle tous les autres sites mais une tendance à la grandeur, à l'imposition d'un bâtiment écrasant se détachant fortement de son environnement immédiat est visible et a suscité la même polémique de la part des riverains, des pouvoirs publics et de la presse que lors de la construction des premiers Kinepolis. Cette conception architecturale diffère totalement de celle du cinéma de quartier, le Ciné Centre $^{1}$, qui est totalement intégré dans une maison se démarquant peu des immeubles environnants.

\section{La décoration intérieure et les activités}

La décoration intérieure diffère totalement dans les deux chaînes. Celle d'UGC est sobre, froide, moderne, propre et très marquée par l'identité visuelle du groupe. Le moindre détail est pensé stratégiquement même si aucun site n'atteint le même stade de réalisation du nouveau concept UGC. L'esthétique de la chaîne est mise en exergue au détriment du merchandising des films projetés, même si l'intérieur des trois cinémas est notamment agrémenté de photographies d'acteurs. Nous assistons là à une mise en évidence des acteurs reconnus pour leur métier davantage que pour les films réalisés. Les cinémas sont les lieux de déroulement d'événements VIP, non accessibles au public. Une légère diversification des services commence mais la chaîne préfère s'intégrer dans des projets plus larges tels que le "Ciné Cité". Jusqu'à présent, les concepteurs étaient soucieux de préserver des traces du passé provenant des anciens cinémas mais le concept est aujourd'hui en pleine évolution et cette tendance s'amenuise avec la construction de complexes sur des sites vierges.

1 Intégré dans un contexte semi-urbain à Rixensart, le Ciné Centre est un cinéma de quartier qui a vu le jour en 1930 et a été relancé en 1990. Son but est d'allier le confort au charme d'une ancienne salle. Le propriétaire s'occupe à la fois de la caisse, de la projection du film et de la décoration du lieu. Sa stratégie de choix de films vise des produits porteurs et d'autres plus spécialisés destinés à un public d'amoureux du cinéma. Le Ciné Centre a reçu le Coq d'or récompensant le cinéma le plus méritant de la Communauté française. 
Kinepolis Group présente une décoration fragmentée et brouillonne qui diffère d'un site à l'autre en fonction du manager du complexe. Les mêmes objets sont présents mais leur place dans le décor n'est pas stratégiquement pensée, contrairement à ce que l'on observe à l'UGC. Le merchandising des films et les sponsors envahissent l'espace intérieur. Ces partenaires économiques peuvent utiliser un endroit du cinéma pour exposer leurs produits ou les mettre à disposition du public. De nombreux événements s'y déroulent également. L'esthétique de la chaîne semble passer au second plan, "envahie" par celles des alliés économiques et des films proposés. Et cette tendance s'accroît avec le temps, comme la dégradation progressive des locaux. La qualité de projection et les services proposés sont plus importants que l'identité visuelle qui peut varier d'un site à 1'autre. Cette conception s'oppose à celle privilégiant la permanence et la nostalgie qui se dégage de l'aménagement intérieur du Ciné Centre mais elle s'en rapproche par l'éclectisme de la décoration et un certain amateurisme.

\section{La relation entre personnel et clientèle}

La notion d'accueil est plus développée à Kinepolis qu'à UGC. Cela se traduit par un stand destiné spécifiquement à répondre aux questions de la clientèle. D'autres indices nous permettent de croire à l'importance de la relation avec les spectateurs, dont un badge au nom de l'employé et un mot de bienvenue destiné à connaître la langue du client pour ensuite l'orienter dans le complexe. Ces éléments donnent l'impression d'une relation privilégiée avec le personnel. Les bureaux de Metropolis, visibles de la clientèle, accentuent ce sentiment d'ouverture, de transparence et de proximité entre l'organisation et les spectateurs. Le responsable de communication est facilement joignable et n'hésite pas à donner des informations, le personnel également. Comme sa décoration, la relation du personnel de l'UGC avec le public est plus froide, formelle et peu de renseignements sont fournis.

Malgré ces indices de relation au consommateur relevant de l'économie familiale ${ }^{1}$ avec présence du don (objets publicitaires) pour Kinepolis, c'est plutôt une relation marchande qui est instaurée. UGC

1 V. CARAyol, "Du clinquant au sensible, la modestie mise en scène", Recherches en communication, $\mathrm{n}^{\circ}$ 17, Louvain-la-Neuve, 2002, pp. 95-112. 
est plus clair dans cette démarche puisqu'il n'y a aucune ambiguïté sur le type de relation qui est établie entre la chaîne et les clients : le public sait qu'il a affaire à une relation de type marchand totalement opposée à celle chaleureuse proposée par le propriétaire du Ciné Centre.

\section{Les dispositifs de communication publicitaire}

Certaines différences notables existent dans la façon qu'ont les deux chaînes de se présenter au public.

Avec l'ouverture de son nouveau site à Louvain-la-Neuve, UGC s'est lancé dans une large campagne de publicité. La couleur bleue, soulignée d'un peu de bordeaux, rappelle les coloris mis en évidence dans les locaux et dans le logo-donc dans l'identité visuelle de la chaîne. Des renseignements pratiques, la présentation de nouveaux services et la mise en valeur des qualités du complexe sont les éléments avancés par la chaîne.

Le slogan est également très révélateur : Le cinéma comme vous l'avez rêvé. Il fait référence à l'imaginaire du public, à ses attentes et à la fonction d'évasion remplie aujourd'hui par les cinémas.

Kinepolis, lui, crée son propre journal, disponible dans ses complexes et composé de reportages sur les films, de concours et de publicités sur ses services. Ses nombreux partenaires économiques y apparaissent. Outre le logo, on y décèle la mise en évidence des quatre critères de sélection de sites, décrits plus haut.

C'est dans les salles que nous retrouvons des publicités audiovisuelles vantant le complexe et ses services. Nous pouvons donc remarquer que le groupe vise un public jeune et la clientèle déjà sur place en vue de les fidéliser. Comme la décoration, les messages publicitaires sont également influencés par le partenariat et le merchandising des films. L'esthétique du Ciné Centre communique, lui, l'amour du propriétaire pour le 7ème art, les dispositifs publicitaires étant tournés essentiellement vers les films projetés ou les grands classiques du cinéma.

\section{Vers une évolution de la stratégie}

À travers des éléments comme l'architecture, la décoration intérieure, les activités développées à l'intérieur des sites, la relation entre 
le personnel et les clients et les dispositifs de communication publicitaire, nous décelons une évolution de la stratégie des chaînes de cinéma. Cette évolution est clairement présentée par Sylvie Declercq dans un article de Media Marketing ${ }^{1}$. Elle y explique que les chaines repensent leurs complexes en "cités de l'audiovisuel et du loisir", véritables plates-formes événementielles où la rencontre est plébiscitée. Cela permet de redynamiser l'offre, de fidéliser le spectateur, d'accroître la notoriété et de rentabiliser les investissements. De plus, les sites ne sont plus uniquement pensés en fonction du client habituel, consommateur de films, mais également en fonction des entreprises qui voudraient y réaliser un événement. Kinepolis évolue vers un concept de "Family Entertainment Centers" véhiculant des valeurs sociales comme la famille, le loisir ou la sécurité, par l'incorporation d'autres organisations au sein de ses sites. La stratégie d'UGC est, elle, d'intégrer ses cinémas dans des projets plus larges, des "Ciné Cités" et implique également de plus en plus d'événementiel par l'organisation de soirées de prestige auxquelles voudraient participer de grands annonceurs. Chaque fête ou festival est une occasion pour créer un événement. UGC et Metropolis ont également développé un centre de communication destiné aux entreprises. Ce dernier propose des services tels que l'organisation de conférences, de réunions, de soirées VIP ou d'avant-premières de films en projection privée.

Cette évolution des chaînes de cinéma peut être rapprochée des considérations de Georges Ritzer ${ }^{2}$ qui voit dans l'extension du nombre de lieux de grande fréquentation et de grande chalandise, une tendance lourde de l'évolution de la consommation actuelle. On voit apparaître des "lieux mixtes, combinant une finalité particulière propre à une finalité de type davantage généraliste. Cette dernière permet aux personnes qui les fréquentent non seulement d'être rassemblées dans un milieu pour une raison spécifique, mais aussi d'occuper leurs temps libres pour satisfaire leurs besoins de consommateurs" 3 . Dans un contexte de mondialisation croissante, ces lieux deviennent des méga-complexes de la consommation, de loisirs et de

I S. DeClercQ, "Des salles de moins en moins obscures", Média Marketing, n ${ }^{\circ}$ 158, mai-juin 2000, pp. 26-28.

2 A. GRYSPEERDT, "En quoi la mondialisation affecte-t-elle la relation des entreprises avec les consommateurs ?", Les défis de la globalisation, Louvain-la-Neuve, Presses Universitaires de Louvain, 2001, pp. 501-514.

3 Ibid., p. 503. 
culture qui permettent de réenchanter un monde désenchanté, qualifiés par Ritzer de cathédrales de la consommation.

Si cette tendance peut être observée pour Kinepolis (architecture monumentale, possibilité pour le public de s'adonner à des activités très diverses et de bénéficier de multiples services -jouer à l'ordinateur, chercher du travail, envoyer des messages via internet, faire garder ses enfants, organiser des événements...), nous pouvons trouver des traces dans les cinémas UGC annonçant une évolution dans cette voie. En effet, celui d'Anvers est intégré dans un centre qui regroupe restaurants, supermarché et centre de fitness. Le public est encouragé à mêler ces activités (la philosophie des quatre " $F$ " : fun, food, film, fitness). Le cinéma UGC Louvain-la-Neuve, qui représente la stratégie actuelle du groupe, va, lui, être intégré dans un projet de "Ciné Cité", mêlant loisirs, Horeca et activités audiovisuelles. Si les cinémas Kinepolis correspondent déjà, grâce aux actions des divers sponsors, aux "cathédrales de la consommation", UGC s'allie avec des organismes déjà existants (ou qui existeront...) à proximité du cinéma pour élargir ses activités et ses services. Ces évolutions s'opposent à la permanence du cinéma de quartier Ciné Centre.

De par certains éléments de leur esthétique -leur situation "isolée", leur architecture monumentale, leurs façades vitrées permettant les jeux de lumière...- les bâtiments du Kinepolis peuvent réunir les "conditions de l'évasion" propres à d'autres lieux de loisirs analysés par Elizabeth Vercher'. Le public se rendant à Kinepolis quitte le monde concret pour pénétrer dans un univers recentré et clos sur la satisfaction de son désir. Tout y est fourni en abondance pour ne pas lui donner l'envie de sortir. Les UGC présentent également certaines conditions de l'évasion: l'UGC Louvain-la-Neuve, dont l'architecture diffère des bâtiments de la ville, va donner l'impression au public de pénétrer dans un monde "autre"; le lieu est balisé par des panneaux d'indication qui optimisent la conduite du client vers son objet de satisfaction (le café, les salles...).

Nous pouvons encore rapprocher ces constatations sur les deux chaînes des analyses des shopping centers faites par Axel Gryspeerdt ${ }^{2}$. Ici aussi, on retrouve deux éléments : le mouvement (le public se déplaçant sur des passerelles, des rampes...) et la lumière (baies

\footnotetext{
1 E. VerChER, "Les bandits-manchots, Mickey et les G.O. ou l'esthétique des loisirs post-modernes", Recherche en communication, $n^{\circ}$ 18, Louvain-la-Neuve, 2002.

2 A. GRYSPEERDT, op cit., pp. 504-505.
} 
vitrées, faisceaux de lumières...) qui accentuent la nature attractive et enchanteresse des cinémas. Le public, ainsi attiré par ces lieux de loisirs, s'adonne à de plus en plus d'activités annexes à la simple vision d'un film (donc "consomme" davantage) et participe alors au "ré-enchantement du monde", selon l'expression de Ritzer!.

\section{Une conception commerciale du cinéma}

L'analyse de l'esthétique des cinémas UGC et Kinepolis nous amène à percevoir que ces chaînes privilégient une conception du cinéma principalement commerciale et cela selon deux axes : la programmation, qui est marquée par le peu de films d'art et d'essai et par la grande présence de films hollywoodiens, et la vision même de leur activité (projection de films), qui tend à devenir moins centrale (contrairement aux cinémas de quartier) et qui est associée à d'autres occupations. Nous notons toutefois que cette conception est privilégiée par Kinepolis plus que par UGC qui, pour l'instant, ne semble pas s'y abandonner totalement.

De produit culturel, le cinéma se réduit donc aujourd'hui à un simple loisir, objet d'une "consommation théâtralisée", selon les termes de Michel Maffesoli².

\section{L'esthétique organisationnelle comme processus de création}

Au vu des résultats de nos recherches, nous découvrons qu'il est très difficile de formuler une définition de l'esthétique organisationnelle de chaque groupe puisque celle-ci est changeante, en cours de concrétisation et en pleine évolution selon les chaînes. Malgré tout, nous avons pu observer un certain nombre de facteurs qui influencent l'esthétique de toute organisation. Tous ces éléments ont un impact plus ou moins important sur la création de ce phénomène. Il s'agit de facteurs extérieurs à l'organisation, qui varient selon des contraintes spatio-temporelles, tels que les lois en vigueur, les desiderata des pouvoirs publics, l'actualité, le public, la concurrence, la culture

1 Ibid.

2 M. MAFFESOLI, La contemplation du monde, figures du style communautaire, Paris, Grasset, 1993, p. 113. 
régionale, nationale ou internationale... L'organisation est obligée de prendre en considération ces éléments externes. Ensuite, il y a tous les facteurs internes à l'organisme qui sont l'aboutissement de ses multiples choix : le choix de son personnel, de son identité, de l'image qu'elle veut diffuser, de ses partenariats, du produit qu'elle commercialise, de sa politique organisationnelle et managériale... Ici encore, les facteurs sont nombreux et ont tous une influence plus ou moins importante sur le concept que nous étudions. En effet, selon divers facteurs hétéronomes et autonomes liés à l'organisation, tout un processus de création est mis en œuvre, de manière plus ou moins stratégique, pour se réaliser concrètement dans des manifestations tangibles comme l'architecture, la décoration intérieure, l'uniforme, le logo, l'habitation du lieu, les éléments olfactifs et auditifs... qui, ensemble, forment l'esthétique de l'organisation.

L'esthétique organisationnelle est donc le résultat d'un processus de création, plus ou moins conscient, influencé par des contraintes externes et internes, qui se manifeste dans des phénomènes concrets divers dont le public fait l'expérience de façon cognitive et sensible. L'esthétique et l'expérience du public rétroagissent ensuite à des degrés différents sur ces facteurs. Nous avons reproduit ce processus dans le schéma de la page.

Si nous comparons cette définition avec celle que nous avons présentée en début de texte et sur laquelle nous nous sommes basées, nous pouvons constater une évolution, un affinement de cette dernière. Nous décrivions sa manifestation concrète tandis que par cette recherche, nous comprenons comment ce phénomène est créé -même si cette création évolue sans cesse puisque ce concept est luimême composé d'éléments mouvants. De plus, il a un impact sur l'expérience sensible et intelligible de l'organisation qu'ont quatre sortes d'actants subjectifs puisque humains : les dirigeants, le personnel, les clients et les médias. Ceux-ci, à leur tour, influencent l'esthétique: les deux premiers ont un rôle plus direct dans l'évolution de ce phénomène, les autres l'influencent de par leur rôle dans le succès d'une entreprise. 


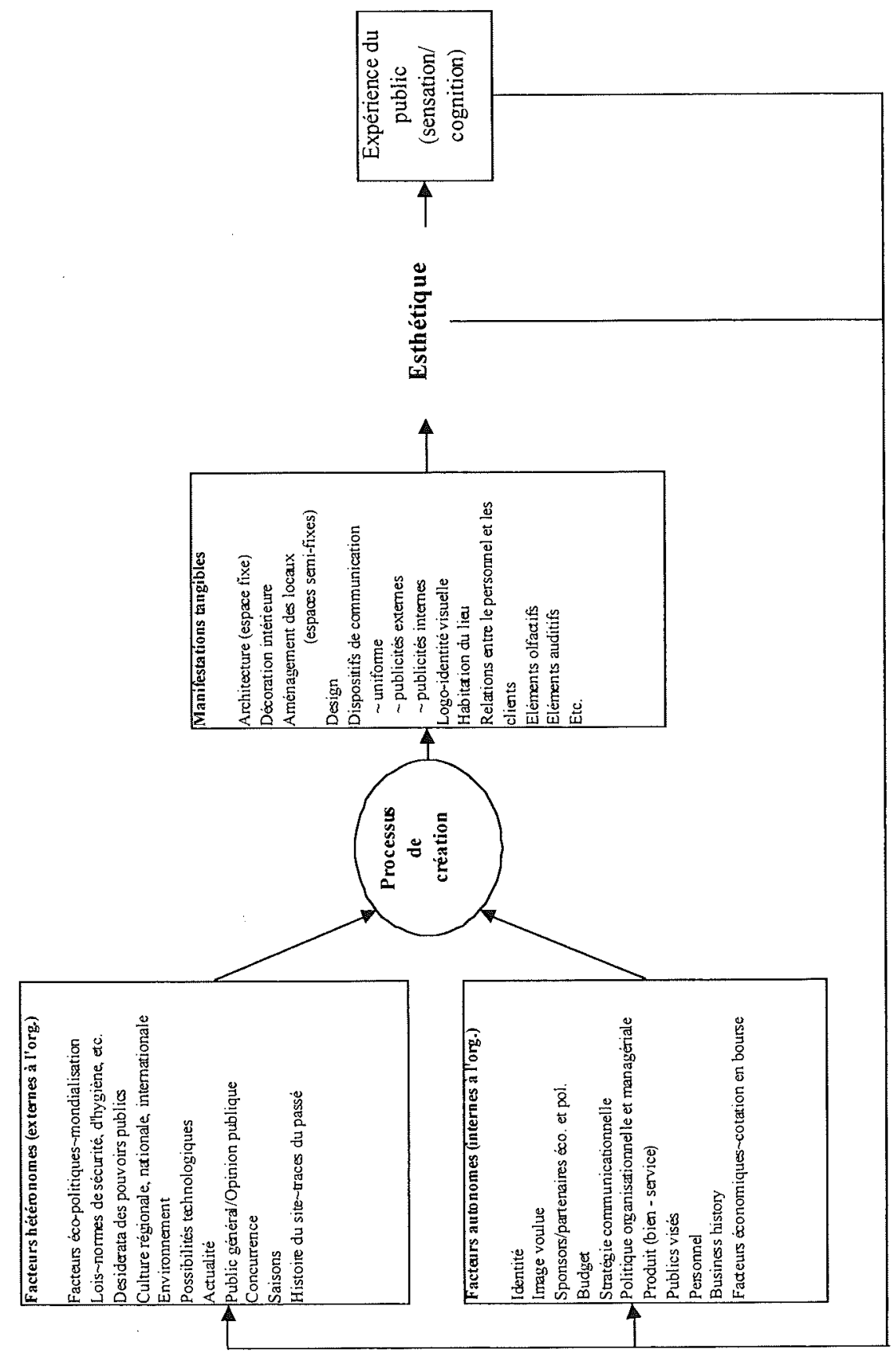


Si nous partons du postulat que notre schéma explique comment ce phénomène se produit et se concrétise, nous devons maintenant nous poser les questions suivantes : pourquoi ce processus peut-il être stratégiquement mis en œuvre par les organisations ? Dans quel but?

Nous avons déjà donné des éléments de réponse à ces questions précédemment, en comparant les deux chaînes. Si nous généralisons à toute organisation et envisageons l'esthétique comme production de l'organisation, nous pouvons dire que l'esthétique vue par les dirigeants permet de faire passer un message aux clients, d'expliquer de façon muette la conception qu'a l'organisme de son produit, de son travail et de son rapport aux autres, qu'ils soient concurrents, clients, employés ou journalistes... Ainsi l'organisation se met en scène pour mieux se vendre mais aussi pour tenter de prouver qu'elle n'est pas là uniquement pour échanger un bien ou un service contre de l'argent mais pour apporter à cet échange quelque chose de plus, d'immatériel. Ce quelque chose diffère selon les buts organisationnels. Dans le cas des lieux de loisirs, dont les cinémas font partie, il s'agit du rêve, de l'évasion et du réenchantement du monde quotidien.

Mais il faut également envisager l'esthétique comme phénomène lié à la perception du public. En effet, l'objectif organisationnel n'est pas toujours atteint car le message propagé peut ne pas avoir été perçu par le public de la façon souhaitée, ou ne pas correspondre aux attentes de celui-ci. Dans ce cas, les récepteurs du message peuvent également agir sur celui-ci. Même si l'organisation pense stratégiquement à la meilleure façon de combiner les facteurs externes et internes, le résultat risque d'être différent de ce qu'elle a imaginé, tout comme l'œuvre échappe à son créateur. L'esthétique mêle l'intelligible au sensible en une gestalt, et échappera toujours à la volonté de ses concepteurs.

\section{En guise de conclusion}

Le concept d'esthétique des organisations est apparu récemment dans le domaine de la recherche en communication organisationnelle. Les résultats de notre démarche nous ont permis, d'une part, de déceler les phénomènes de communication liés à l'expérience esthétique (les "évidences esthétiques") et, d'autre part, de confronter ceux-ci aux politiques de communication mises en œuvre par les chaînes. Nous avons pu constater certaines inadéquations entre nos observa- 
tions et les stratégies exprimées par les responsables de communication. Ainsi, les sites de Kinepolis présentent des caractéristiques esthétiques fragmentées et souvent différentes alors que le groupe prône une politique de communication claire et unique. UGC présente, selon nous, certains éléments caractéristiques des complexes cinématographiques envisagés comme lieux de loisirs et de consommation, mais prône une conception culturelle de ces mêmes lieux.

Au terme de notre recherche, il est difficile de décrire succinctement l'esthétique de chacune des chaînes. La réalité du phénomène esthétique est complexe et ce n'est qu'en comparant les deux chaînes entre elles et, ensuite, avec le cinéma de quartier que nous sommes parvenues à dégager les grandes tendances esthétiques de nos objets d'étude.

À un premier niveau de comparaison, nous pouvons conclure que l'esthétique du cinéma de quartier est une "invitation" au monde du cinéma, au monde du film projeté perçu comme produit culturel, tandis que l'esthétique des deux chaînes de cinéma constitue une "invitation" à un monde propre, à un univers particulier, différent de celui du produit cinématographique.

$\grave{A}$ un second niveau de comparaison, nous avançons que les univers dans lesquels nous plongent l'esthétique d'UGC et celle de Kinepolis sont très différents. Pour UGC, l'esthétique organisationnelle sobre et calculée est dominante. L'organisme lui-même est mis en avant à l'extérieur comme à l'intérieur des bâtiments. L'esthétique de Kinepolis est, elle, plutôt commerciale ; il s'agit d'une esthétique du merchandising liée aux films, hollywoodiens pour la plupart.

Notre étude nous a permis de relever les différents éléments qui influencent le processus de création menant à des manifestations tangibles de l'esthétique. Plus que définir véritablement le concept d'esthétique organisationnelle, nous avons pu découvrir comment une organisation en arrivait à présenter une esthétique particulière au public, qui en trouve ainsi son expérience sensible et cognitive affectée. 